\title{
15-Lipoxygenase is a component of the mammalian sperm cytoplasmic droplet
}

\author{
K A Fischer ${ }^{1}$, K Van Leyen ${ }^{3}$, K W Lovercamp ${ }^{1}$, G Manandhar ${ }^{1}$, M Sutovsky ${ }^{1}$, D Feng ${ }^{1}$, \\ $\mathrm{T}_{\text {Safranski }}{ }^{1}$ and P Sutovsky ${ }^{1,2}$ \\ ${ }^{1}$ Division of Animal Sciences and ${ }^{2}$ Department of Obstetrics and Gynecology, University of Missouri, Columbia, \\ Missouri, USA and ${ }^{3}$ Neuroprotection Research Laboratory, Department of Radiology, Massachusetts General \\ Hospital, Charlestown, Massachusetts, USA
}

Correspondence should be addressed to P Sutovsky, Division of Animal Science and Department of Clinical Obstetrics and Gynecology, University of Missouri, Columbia, S141 ASRC, 920 East Campus Drive, Columbia, Missouri 65211-5300, USA; Email: sutovskyp@missouri.edu

\begin{abstract}
Lipoxygenases (LOXs) are a family of enzymes capable of peroxidizing phospholipids. A member of the LOX family of enzymes, 15-LOX, participates in the degradation of mitochondria and other organelles within differentiating red blood cells, the reticulocytes. The present study provides biochemical and immunocytochemical evidence for the presence of 15-LOX in the sperm cytoplasmic droplet (CD). Testicular, epididymal and ejaculated spermatozoa were evaluated for the presence of 15-LOX using an affinity-purified immune serum raised against a synthetic peptide corresponding to the C-terminal sequence of rabbit reticulocyte 15-LOX. Western blotting revealed an appropriate single band of $\sim 81 \mathrm{kDa}$ in boar spermatozoa but not in boar seminal plasma. When ejaculated boar spermatozoa were subjected to separation on a $45 / 90 \%$ Percoll gradient, 15-LOX co-migrated with the immotile sperm and cellular debris/CD fractions, but not with the motile sperm fraction containing morphologically normal spermatozoa without CDs. Varied levels of 15-LOX were expressed in ejaculated sperm samples from boars with varied semen quality. By immunofluorescence, prominent 15-LOX immunoreactivity was found within the residual body in the testis and within the CDs from caput, corpus and cauda epididymal and ejaculated spermatozoa. Components of the ubiquitin-dependent proteolytic pathway, which is thought to facilitate both spermiogenesis and reticulocyte organelle degradation, were also detected in the sperm $\mathrm{CD}$. These included ubiquitin, the ubiquitin-conjugating enzyme E2, the ubiquitin C-terminal hydrolase PGP 9.5, and various 20 proteasomal core subunits of the $\alpha$ - and $\beta$-type. The 15-LOX and various components of the ubiquitin-proteasome pathway were also detected in sperm CDs of other mammalian species, including the human, mouse, stallion and wild babirusa boar. We conclude that 15-LOX is prominently present in the mammalian sperm $C D$ and thus may contribute to spermiogenesis, $C D$ function or $C D$ removal.

Reproduction (2005) 130 213-222
\end{abstract}

\section{Introduction}

Spermatogenesis ensures the production of spermatozoa capable of acquiring progressive motility and the ability to recognize, bind to and fuse with an oocyte. This is achieved in part by the reduction of sperm cytoplasmic volume and organelle pool during spermiogenesis in the testis. The removal of the last portion of male germ cell cytoplasm, the sperm cytoplasmic droplet (CD), puts a final touch on the process of sperm morphogenesis. The sperm $\mathrm{CD}$ is composed of cytosol and organelle-derived membranes, which are thought to be the remnants of Golgi and possibly other spermatid organelles degraded during the final stages of spermiogenesis (Oko et al. 1993). The CD of mammalian spermatozoa is approximately $2 \mu \mathrm{m}$ in diameter (Kaplan et al. 1984), and in most mammalian species is believed to be removed from the sperm midpiece during epididymal passage or after ejaculation. Although observed to a varied degree in most other mammals, CD retention on the sperm midpiece is the most frequent abnormality in ejaculated boar spermatozoa in boars of poor fertility. In this species, shed CDs accumulate in the ejaculate in the form of cellular debris. The presence of CDs either on the sperm midpiece (Waberski et al. 1994, Thundathil et al. 2001) or detached in the ejaculate (Aitken \& Clarkson 1988) could reduce the fertilizing ability of the ejaculated boar spermatozoa. While the CDs retained on the sperm midpiece can be accounted for using conventional 
light-microscopical semen analysis, it is difficult to quantify the amount of free, detached CDs in boar semen. This presents a challenge in the swine industry, wherein over $70 \%$ of sows are bred using artificial insemination (National Animal Health Monitoring System Survey 2000), and mixing of ejaculates from several boars with varied sperm parameters is a common practice. It is thus desirable to identify new biomarkers that would allow development of an objective, automated assay for evaluation of total CD content in boar semen (reviewed by Althouse 1998).

Organelle degradation pathways were studied in detail during the differentiation of precursor red blood cells, the reticulocytes, in which the mitochondria, Golgi and other cellular organelles are abolished. The components of two distinct, synergistic pathways, the lipoxygenase (LOX) and the ubiquitin-proteasome pathways, were identified as major players in the process of reticulocyte organelle degradation. LOXs are a family of enzymes responsible for peroxidation of lipids such as arachidonic acid. 15-LOX has been shown to peroxidize lipids in organelle membranes during reticulocyte differentiation (Schewe et al. 1975). LOXs have also been implicated in the induction of sperm acrosomal exocytosis during mammalian fertilization (Oliw \& Sprecher 1989, Lax et al. 1990). Ubiquitin, a small chaperone protein, is common to all eukaryotic cells and plays an important role in proteasomal and lysosomal protein degradation. Ubiquitin is thought to facilitate proteolysis and organelle degradation during reticulocyte differentiation (Rapoport \& Schewe 1986, Wefes et al. 1995). There is an indication that ubiquitin acts in synergy with the 15-LOX pathway during mitochondrial membrane degradation in the reticulocytes (Dubiel \& Rapoport 1989, van Leyen et al. 1998). Although the respective LOX and ubiquitin pathways may not intersect directly, it is possible that they function in conjunction during organelle degradation. Similarly to reticulocyte differentiation, spermiogenesis relies in part on the ubiquitin system for organelle degradation and proteolysis (reviewed by Escalier 2003, Sutovsky 2003). Ubiquitin is also present in defective mammalian epididymal and ejaculated spermatozoa (Sutovsky et al. 2001). Considering the requirement of ubiquitin-dependent proteolysis during spermatid elongation (Baarends et al. 1999, Escalier 2003, Sutovsky 2003), it is possible to envision a parallel relationship between 15-LOX and ubiquitin in reticulocyte maturation and spermatid elongation. Such synergy between the LOX and ubiquitin pathways could effectively release and degrade the respective lipid and protein components of spermatid organelle membranes without damaging the integrity of the spermatid plasma membrane. The present study provides direct biochemical and immunocytochemical evidence of 15-LOX expression in the spermatid residual body and the CD of epididymal and ejaculated spermatozoa, consistent with a possible role of 15-LOX in spermiogenesis and epididymal sperm maturation.

\section{Materials and Methods}

All animal procedures were conducted in accordance with approved Animal Care and Use Commitee protocols.

\section{Boar semen samples}

Boar ejaculates were obtained by the gloved-hand technique from crossbred and Large White boars at the University of Missouri swine farm. Eight boars used in the present studies had histories of varied sperm morphology and high and low frequency of CD retention. All boars were between 9 months and 2 years of age at the time of collection. Samples were collected as needed, with a minimum 3 day resting period between ejaculates and a maximum of 10 days between collections. Six other boars were used for sperm and tissue isolation from the testis and epididymis.

\section{Epididymal and testicular spermatozoa}

Boars from a commercial boar stud $(n=1)$ and the University of Missouri's swine herd $(n=5)$ were killed following the approved ACUC protocols at the boar stud and the University of Missouri Veterinary Medical Diagnostic Laboratory respectively. Tissues (caput, corpus and cauda epididymis) from both the left and right side of the reproductive tract were collected. Sperm samples from all four compartments were obtained by repeated, gentle mincing of the tissue to allow sperm cells to flow into Sperm TL medium with polyvinyl pyrrolidone. Spermatozoa were passed through a $105 \mu \mathrm{m}$ SpectraMesh macroporous filter (Spectrum Medical Industries, Inc., Houston, TX, USA) and centrifuged at $500 \mathrm{~g}$ for $5 \mathrm{~min}$ to collect a sperm pellet. The pellet was diluted with Sperm TL medium and sperm morphology was assessed (see Semen Evaluation subsection below).

\section{Semen evaluation}

High-resolution differential interference contrast (DIC) light microscopy was used for semen evaluation to attain high accuracy superior to that of routine field semen analysis, which typically employs microscopes with $\times 20$ or $\times 40$ magnification lenses and simple bright field illumination. Semen evaluation was always performed on fresh samples, never after freeze-thaw. Spermatozoa were photographed under DIC using a $\times 100$ infinity-corrected planar apochromatic lens of a Nikon Eclipse E800 microscope (Nikon, Inc., Melville, NY, USA) equipped with a CoolSnap HQ CCD device (Roper Scientific, Tucson, AZ, USA) operated by MetaMorph software. Recorded images were evaluated on a $19 \mathrm{inch} / 48 \mathrm{~cm}$, high-resolution, flat computer screen. At least 100 spermatozoa from each sample were randomly selected for morphological evaluation and classified as having a normal morphology, a proximal $C D$, a distal $C D$, or other type of abnormal morphology not due to the presence of a CD (sperm head 
and/or sperm tail malformations). While some authors believe that sperm CDs in epididymal or ejaculated spermatozoa may play a functional role in sperm maturation and migration, we considered all types of sperm CDs an abnormality in cauda epididymal and ejaculated spermatozoa for the purpose of morphological evaluation. Spermatozoa were considered to have a proximal CD if the $\mathrm{CD}$ was present at the connecting piece or in the anterior half of the midpiece region. Spermatozoa were considered to have a distal CD if the CD was present at the annulus or in the posterior half of the midpiece region. Spermatozoa were considered to have other types of abnormal morphology if they had coiled tails, misshapen heads, misaligned head-midpiece connections or missing heads/tails.

\section{Sperm processing}

Fresh semen samples were centrifuged at $500 \boldsymbol{g}$ for $8 \mathrm{~min}$. Seminal plasma was frozen at $-20^{\circ} \mathrm{C}$ and the sperm pellet was divided; one part of it stored at $-20^{\circ} \mathrm{C}$ and the other part fixed in $2 \%$ formaldehyde for $40 \mathrm{~min}$ on lysinecoated coverslips.

\section{Semen fractionation on Percoll gradients}

Raw, fresh sperm samples were liquefied and centrifuged at $500 \mathrm{~g}$ for $8 \mathrm{~min}$ to form a sperm pellet. A 45/90\% discontinuous Percoll gradient was prepared using $2 \mathrm{ml} 45 \%$ Percoll (1 ml 90\% Percoll and $1 \mathrm{ml}$ Sperm TL medium with BSA) and $2 \mathrm{ml} 90 \%$ Percoll. One milliliter of the sperm pellet was carefully overlaid on top of the Percoll gradient and centrifuged for $5 \mathrm{~min}$ at $1000 \mathrm{~g}$. Spermatozoa were isolated from the individual fractions, and at least 100 spermatozoa per sample per fraction were randomly evaluated and classified according to parameters established (see Sperm Evaluation subsection above). Separated spermatozoa were washed twice through $8 \mathrm{ml}$ protein-free Sperm TL medium with polyvinyl pyrrolidone at $500 \mathbf{g}$ for $8 \mathrm{~min}$. The sperm pellets were divided and either placed in a $-20{ }^{\circ} \mathrm{C}$ freezer or fixed in $2 \%$ formaldehyde for $40 \mathrm{~min}$ on lysine-coated coverslips.

\section{Antibodies}

Rabbit anti-15-LOX serum was raised and affinity purified against a synthetic peptide corresponding to a C-terminal domain of rabbit reticulocyte 15-LOX (YLRPSIVENSVAI; van Leyen et al. 1998; diluted 1/100 for immunofluorescence, $1 / 2000$ for Western blotting). Mouse antibodies were raised against human recombinant ubiquitin (KM691; Kamyia Biomedical, Seattle, WA, USA; diluted $1 / 100$ ) and the rabbit serum against ubiquitin C-terminal hydrolase PGP 9.5 (AB1761; Chemicon International, Temecula, CA, USA; diluted 1/100). Rabbit ubiquitin-conjugating enzyme E2 serum (diluted 1/100) and a proteasomal subunit antibody sampler (all diluted 1/100), containing rabbit immune sera recognizing the subunit MECL-1 and a number of other $\alpha$ - and $\beta$-type proteasomal $20 \mathrm{~S}$ core subunits, were purchased from Affinity Research Ltd, Mammhead, UK, represented in the US by Biomol (Plymouth Meeting, PA, USA). Mouse anti- $\beta$-tubulin IgG E7 for Western blotting controls was purchased from the Developmental Studies Hybridoma Bank, maintained by the University of lowa, IA, USA.

Secondary antibodies used in these experiments were: goat anti-rabbit conjugated with fluorescein isothiocyanate or tetramethyl-rhodamine isothiocyanate (GAR IgG-FITC and GAR IgG-TRITC respectively; Zymed, San Francisco, CA, USA, diluted 1/80) and goat anti-rabbit conjugated with horseradish peroxidase (GAR IgG-HRP; Zymed, Lot 00962107, diluted 1/10000). Where applicable, DNA was counterstained with DAPI (4',6-diamidino-2-phenylindole hydrochloride, diluted 1/80).

\section{Immunocytochemistry and immunohistochemistry}

Coverslips with spermatozoa fixed fresh on poly-L-lysine coverslips were permeabilized using PBS with $0.1 \%$ Triton $\mathrm{X}-100$ for $40 \mathrm{~min}$ and washed in 5\% normal goat serum (NGS) in PBS with $0.1 \%$ Triton X-100 (Sutovsky $\mathrm{P}$ et al. 2004). Anti-15-LOX antibody (see Antibodies subsection above) was diluted in $1 \%$ NGS in PBS with $0.1 \%$ Triton X100 and added to spermatozoa for $40 \mathrm{~min}$. After washing in $1 \%$ NGS in PBS with $0.1 \%$ Triton $\mathrm{X}-100$, the secondary antibody, GAR IgG-FITC, and DAPI were diluted in $1 \%$ NGS in PBS with $0.1 \%$ Triton X-100 and added to the coverslips for $1 \mathrm{~h}$. Negative controls were performed by incubation with pre-immune rabbit serum (diluted 1/100), followed by GAR IgG-FITC or GAR IgG-TRITC. Immunohistochemistry on boar testicular and epididymal paraffin tissue sections was performed using a standard protocol (Sutovsky et al. 2001) and probes, reagents and conditions described above. Images were acquired using a CoolSnap HQ CCD camera (Roper Scientific) operated by MetaMorph software (Universal Imaging Corp., Downington, PA, USA), using appropriate epifluorescence filters on a Nikon Eclipse E800 microscope. Images were printed using Adobe Photoshop 5.5 (Adobe Systems, Inc., San Jose, CA, USA).

\section{Western blotting}

Sperm proteins were extracted from frozen sperm samples by adding an extraction buffer containing $50 \mathrm{mM}$ Tris- $\mathrm{HCl}, 20 \mathrm{mM}$ imidazole, $1 \mathrm{mMEDTA}, 1 \mathrm{mM}$ phenylmethylsulfonyl fluoride, Protease Inhibitor Cocktail (Sigma; lot \#102K41) and $5 \mathrm{mM}$ benzamidine $\mathrm{HCl}(\mathrm{pH}$ $6.0)$ to the sperm sample (2 $\mathrm{vol} / \mathrm{vol})$. The sperm solution was ground at maximum speed for $3.5 \mathrm{~min}$ using a pestle attached to a $9.6 \mathrm{~V}$ electric drill (Black and Decker; model no. CD9600). Samples were centrifuged in a cooled microcentrifuge at $4{ }^{\circ} \mathrm{C}$ at $16000 \mathrm{~g}$ for $60 \mathrm{~min}$. Standard Bradford protein estimation was performed using a BioRad Protein Assay kit (Bio-Rad Laboratories, Hercules, 
CA, USA) at $595 \mathrm{~nm}$ wavelength absorbance. In some experiments, protein standardization was confirmed by Western blotting with anti- $\beta$-tubulin antibody E7 (Developmental Studies Hybridoma Bank, University of lowa). Samples were boiled for 5 min with $2 \%$ SDS, $20 \%$ glycerin, $0.05 \%$ bromphenol blue and $5 \% \beta$-mercaptoethanol, and electrophoretically separated on a $10 \%$ Trisglycine gel (BioWhittaker Molecular Applications, Rockland, ME, USA) using 19-30 $\mu$ g protein per sample lane. Kaleidoscope Prestained Standards (Bio-Rad Laboratories) were used as the molecular mass marker. After electrophoresis, proteins were transferred to polyvinylidene fluoride membranes (Immobilon-P Transfer Membranes; Millipore Corp., Bedford, MA, USA) using a wet transfer system (Model \#VEP-2; Owl Scientific, Inc., Woburn, MA, USA). Blots were blocked for $17-30 \mathrm{~h}$ using Blocker Casein in PBS solution (Pierce, Rockford, IL, USA). Anti15-LOX (diluted 1/2000, see Antibodies subsection above) was added to $0.25 \%$ Tris base saline (TBS)-Tween 20 and then incubated with the membrane for $18-28 \mathrm{~h}$. Blots were washed three times in $0.25 \%$ TBS-Tween 20 and then incubated for $1 \mathrm{~h}$ in GAR IgG-HRP. The membrane was washed three times in $0.25 \%$ TBS-Tween 20 and incubated with SuperSignalWest Pico Chemiluminescent Substrate (Pierce). Kodak BioMax Light Film were exposed and developed by using a Kodak M35A X-OMAT Processor. Negative controls were performed using nonimmune rabbit serum.

\section{RNA isolation and RT-PCR}

Total RNA was isolated from 10-30 mg frozen boar testicular and caput, corpus and cauda epididymal tissues or $1.5 \mathrm{ml}$ boar blood using a Qiagen RNeasy Mini RNA isolation kit (Qiagen, Valencia, CA, USA). DNase digestion was used with an RNase Free DNase Set from Qiagen, according to the manufacturer's specifications. The RNA samples were quantified with a NanoDrop spectrophotometer (NanoDrop, Wilmington, DE, USA) at $260 \mathrm{~nm}$ and amplified by a two-step RT-PCR using Oligo-dT primer(15n) (Promega, Madison, WI, USA) in the first step of cDNA synthesis. A Qiagen Sensiscript RT-PCR kit was used for the RT-PCR reaction; $20 \mu \mathrm{l}$ RT mix were incubated at $37^{\circ} \mathrm{C}$ for $90 \mathrm{~min}$. Two microliters of RT-PCR products were used for PCR with gene-specific primers designed to span intron regions, to eliminate false-positive PCR fragments generated from genomic DNA. The primer sequences based on Sus scrofa 15-LOX mRNA sequence, Gene bank accession number NM_213931were: forward primer $5^{\prime}$-GAAGCTGTATCGGTGGGGTA-3' $\left(\mathrm{T}_{\mathrm{m}} 57^{\circ} \mathrm{C}\right)$, reverse primer $5^{\prime}$-GCCACACCAGAAAATCCTGT-3' $\left(\mathrm{T}_{\mathrm{m}}\right.$ $\left.57^{\circ} \mathrm{C}\right)$. The PCR reactions were performed in an Eppendorf Mastercycler (Eppendorf/Brinkmann, Westbury, NY, USA) using an Eppendorf Hot Start PCR kit and $0.5 \mu \mathrm{M}$ forward and reverse primers. The PCR initially started at $94^{\circ} \mathrm{C}$ for $2 \mathrm{~min}$ denaturation, followed by 38 cycles of $94^{\circ} \mathrm{C}$ for $20 \mathrm{~s}, 57^{\circ} \mathrm{C}$ for $10 \mathrm{~s}, 65^{\circ} \mathrm{C}$ for $25 \mathrm{~s}$. Final extension was at $65^{\circ} \mathrm{C}$ for $2 \mathrm{~min}$. The PCR products were resolved on $2 \%$ agarose gel, stained with $0.5 \mu \mathrm{g} / \mathrm{ml}$ ethidium bromide, and photographed on top of a UV light box. The PCR products were sequenced by an Applied Biosystems 3730 DNA Analyzer using Applied Biosystems Prism BigDye Terminator cycle sequencing system (Applied Biosystems, Foster City, CA, USA). The resultant sequences matched the Sus scrofa 15-LOX mRNA sequence from gene bank.

\section{Results}

Semen samples from eight boars of varied fertility, examined by Western blotting with a peptide-specific anti-15-LOX serum, displayed varied quantities of 15-LOX, as determined by variable density of a single band of $\sim 81 \mathrm{kDa}$ (Fig. 1A). This 15-LOX immunoreactivity was mainly contributed by spermatozoa, as the isolated seminal plasma did not display the $81 \mathrm{kDa}$ or other LOXimmunoreactive bands (Fig. 1B). The lack of bands in the negative control indicated the specificity of the antisera (Fig. 1C). The above immune serum recognizes sperm CDs in wild-type mice but not in mutant mice lacking the functional 15-LOX gene (Sutovsky P \& Lovercamp KW; unpublished observations). Expression of the 15-LOX gene in both testis and epididymis was confirmed by RT-PCR (Fig. 1D) and direct sequencing of the amplified product.

To determine whether the 15-LOX immunoreactivity was restricted to CDs or contributed by other sperm components, boar ejaculates were separated on a discontinuous $45 / 90 \%$ Percoll gradient (Fig. 2A and B), resulting in fractions containing separated droplets (45\% supernatant), defective spermatozoa with or without retained CDs (45-90\% interface fraction), and normal, motile spermatozoa without CDs (90\% pellet). While the $90 \%$ pellet fraction was free of 15-LOX, the other two fractions displayed a single, 15-LOX-immunoreactive band of similar density (Fig. 2A). The localization of 15-LOX to the CD alone was confirmed by immunofluorescence microscopy (Fig. 3A-D) and was not observed in sperm samples incubated with pre-immune rabbit serum and an appropriate secondary antibody (Fig. 3I). Interestingly, the components of the ubiquitin-dependent proteolytic pathway were also detected in boar sperm CDs, including ubiquitin (Fig. 3E), ubiquitin-conjugating enzyme E2 (Fig. 3F), ubiquitin C-terminal hydrolase PGP 9.5 (Fig. 3G), and proteasomal core subunits of types $\alpha$ and $\beta$ (Fig. $3 \mathrm{H}$; subunit MECL-1/32i is shown; other proteasomal subunit data not shown). The expression of 15-LOX and its retention in the sperm CD appeared to be conserved as it was also detected in the African wild babyrusa boar (Babyrousa babyrussa; Fig. 3J), human (Fig. 3K), stallion (Fig. 3L), and mouse testicular and epididymal spermatozoa (Fig. 3M and N). The components of the ubiquitin-proteasome pathway, as listed above, were also present in the CDs of these other species (data not shown). 

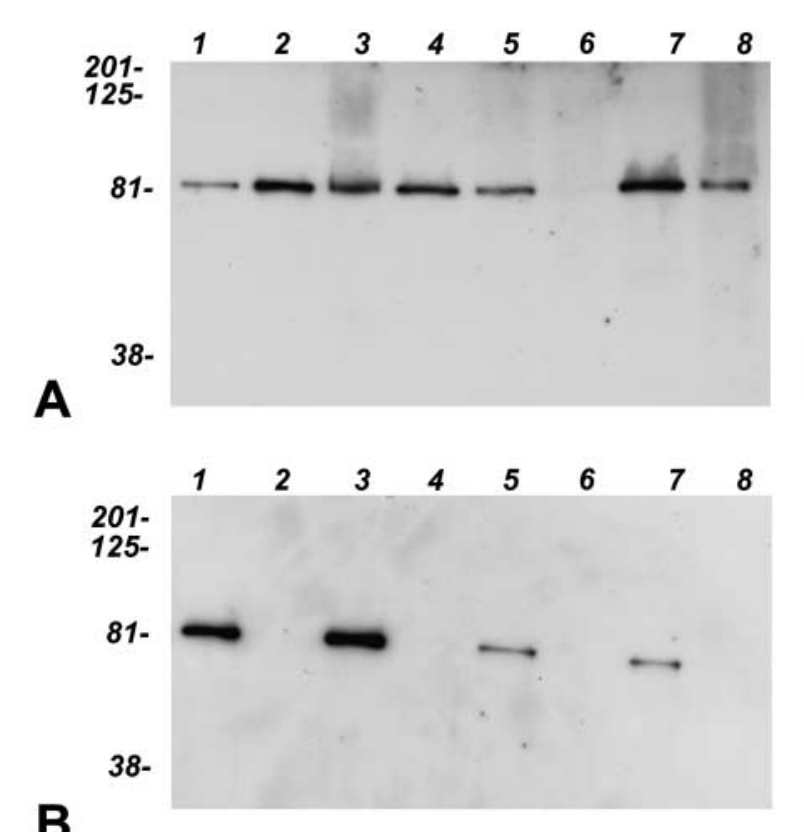

B

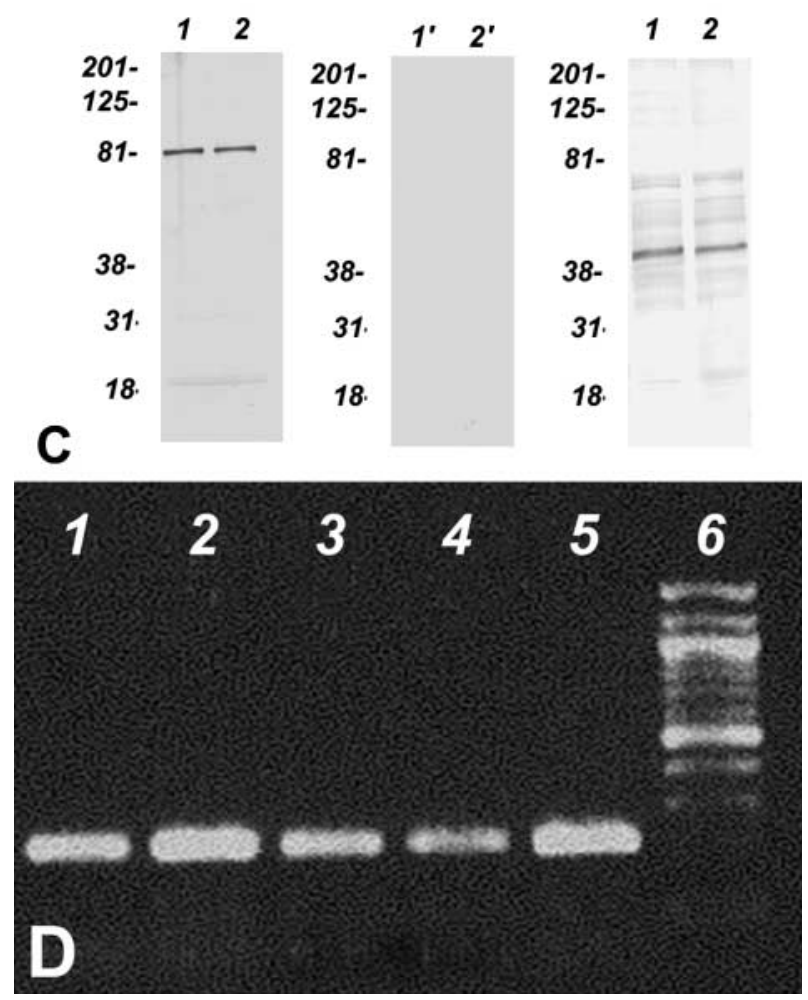

Figure 1 15-LOX immunoreactivity in boar spermatozoa. (A) Western blotting of 15-LOX in semen samples from eight individual boars (lanes 1-8). (B) 15-LOX immunodetection in boar sperm (odd numbered lanes) and seminal plasma (even numbered lanes) of two boars (lanes $1-4=$ boar $\# 1$; lanes $5-8=$ boar \#2) separated at $1000 \mathrm{~g}$ (lanes 1, 2, 5, 6) or $2500 \mathrm{~g}$ centrifugal force (lanes 3, 4, 7, 8). The sperm lanes, but not the seminal plasma lanes, show the 15-LOX band. Protein load was standardized within the blot for both blots by Bradford assay and standardization confirmed by Western blotting with anti-tubulin antibodies. (C) Negative and positive control for Western blotting. An identical pair of boar sperm samples was separated concomitantly on two gels in a dual PAGE chamber. The left gel
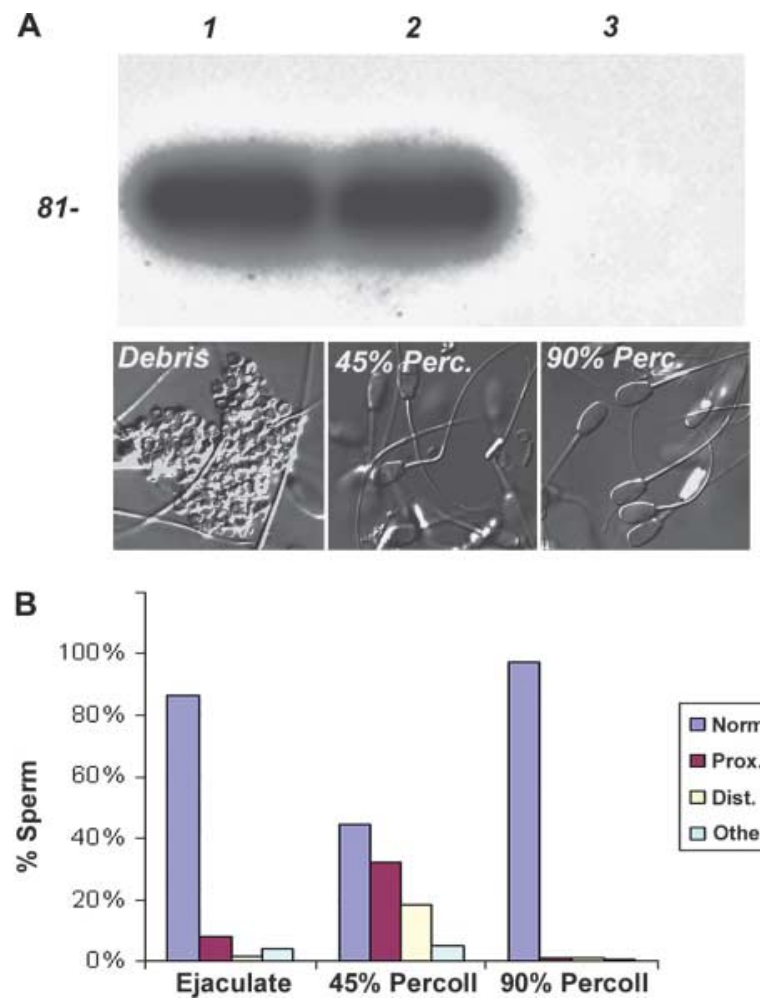

Figure 2 The 15-LOX in boar spermatozoa is confined to the CD. Boar semen was separated on a discontinuous, $45 / 90 \%$ gradient and subjected to Western blotting with anti-15-LOX C-terminal-specific antibody (A). Both the debris with detached CDs (lane 1; 45\% Percoll supernatant) and the CD-bearing sperm fractions (lane 2; 45-90\% Percoll interface) show strong 15-LOX content. The motile sperm fraction (lane 3; sperm pellet which contains mostly spermatozoa without CDs) contains no detected levels of 15-LOX. Inset shows representative DIC images of three respective fractions. (B) Distribution of normal spermatozoa, spermatozoa bearing proximal or distal CDs, and spermatozoa with other morphological abnormalities in the corresponding sample prior to and after Percoll gradient separation. Norm. sp. $=$ sperm with no morphological abnormalities; Prox. $C D=$ spermatozoa with a proximal CD; Dist. $\mathrm{CD}=$ spermatozoa with a distal CD; Other ab. = spermatozoa with abnormalities other than a CD.

Western blotting confirmed the presence of 15-LOX in the spermatozoa isolated from the testis and epididymis (Fig. 4A). Light-microscopic evaluation of the presence of CDs on caput, corpus, and cauda epididymal spermatozoa of six individual boars revealed that the CDs gradually

(lanes 1-2) was sequentially incubated with anti-15 LOX antibody and goat anti-rabbit IgG-HRP. The center gel (lanes $1^{\prime}-2^{\prime}$ ) was sequentially incubated with pre-immune serum and goat anti-rabbit IgG-HRP. The right gel was prepared using the same pair of samples at identical loading and shows anti-tubulin labeling. (D) Expression of the 15-LOX gene in the boar testis (lane 1) and caput, corpus and cauda epididymis (lanes $2-4$ ), as analyzed by RT-PCR. Lane 5 represents porcine erythrocytes (positive control), lane 6 contains molecular mass standards. The identity of PCR products was confirmed by sequencing. 


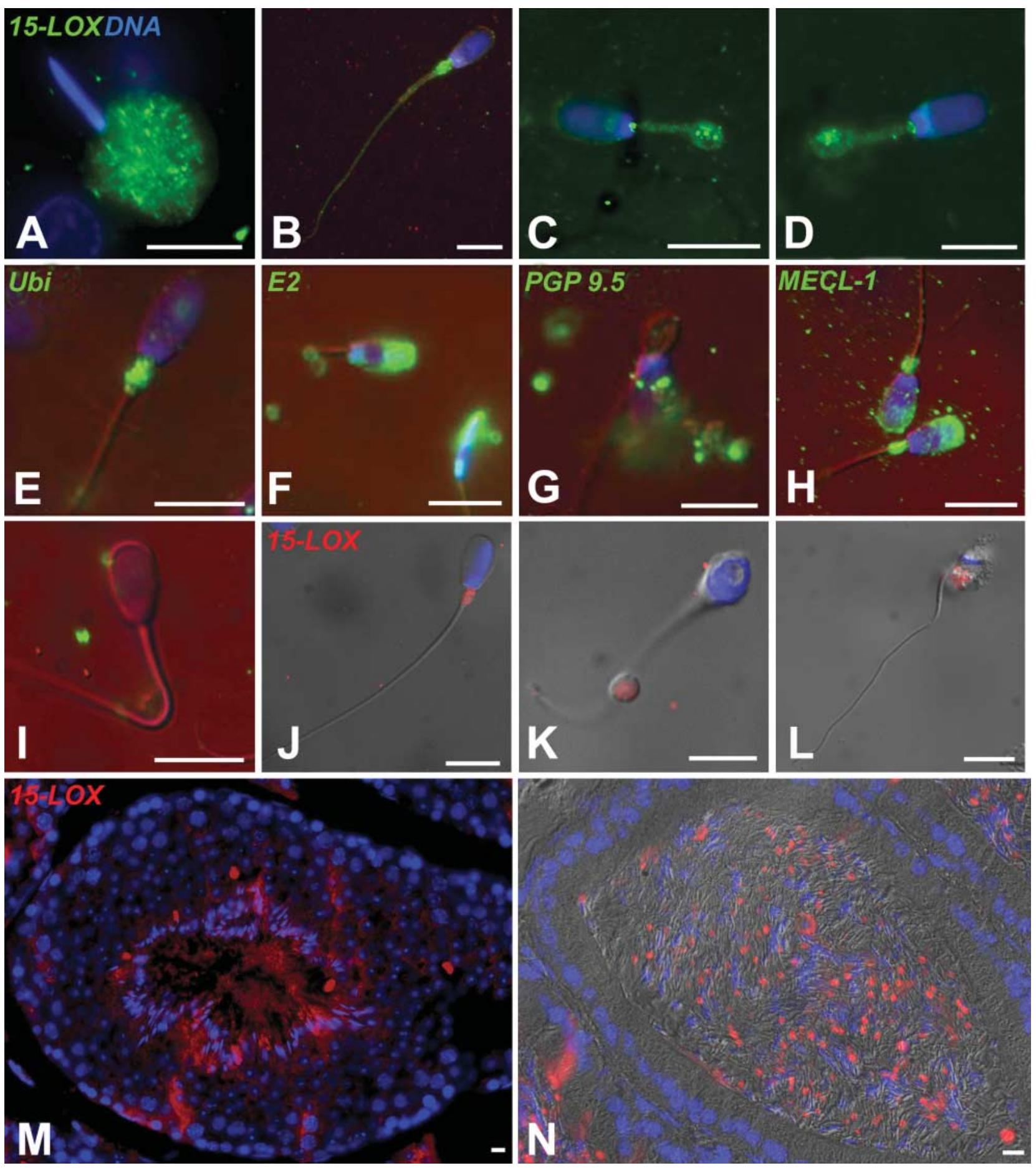

Figure $3 \mathrm{Immunodetection} \mathrm{of} \mathrm{15-LOX} \mathrm{and} \mathrm{ubiquitin-system} \mathrm{enzymes} \mathrm{in} \mathrm{the} \mathrm{mammalian} \mathrm{sperm} \mathrm{CD.} \mathrm{(A-D)} \mathrm{Using} \mathrm{immunofluorescence,} \mathrm{15-LOX}$ (green) colocalized with the cytoplasmic lobe/residual body in a late-stage elongating spermatid in the testis (A) and the migrating CDs in the caput (B), corpus (C) and cauda (D) epididymis. $(\mathrm{E}-\mathrm{H})$ Immunodetection of the proteins of the ubiquitin-proteasome pathway (green) in the boar sperm CD including ubiquitin (E), ubiquitin-conjugating enzyme E2 (F), ubiquitin C-terminal hydrolase PGP 9.5 (G) and proteasomal subunit MECL-1/ß2i (H). (I) Lack of 15-LOX immunolocalization in a distal CD-bearing spermatozoon incubated with pre-immune rabbit serum and an appropriate secondary antibody, serving as a negative control for 15-LOX immunocytochemistry. (J-N) Retention of 15-LOX (red) in the sperm CD of the African wild babyrusa boar (Babyrousa babyrussa; J), man (K) and stallion (L). On paraffin tissue sections, 15-LOX (red) accumulates in the cytoplasmic lobe of elongating spermatids in the mouse testis $(\mathrm{M})$ and in the CD of mouse caput epididymal spermatozoa (N). Scale bars $=10 \mu \mathrm{m}$ 


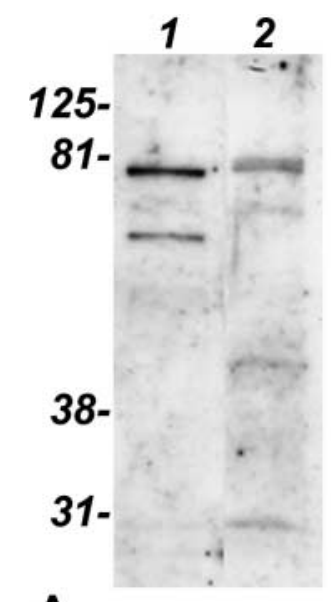

A

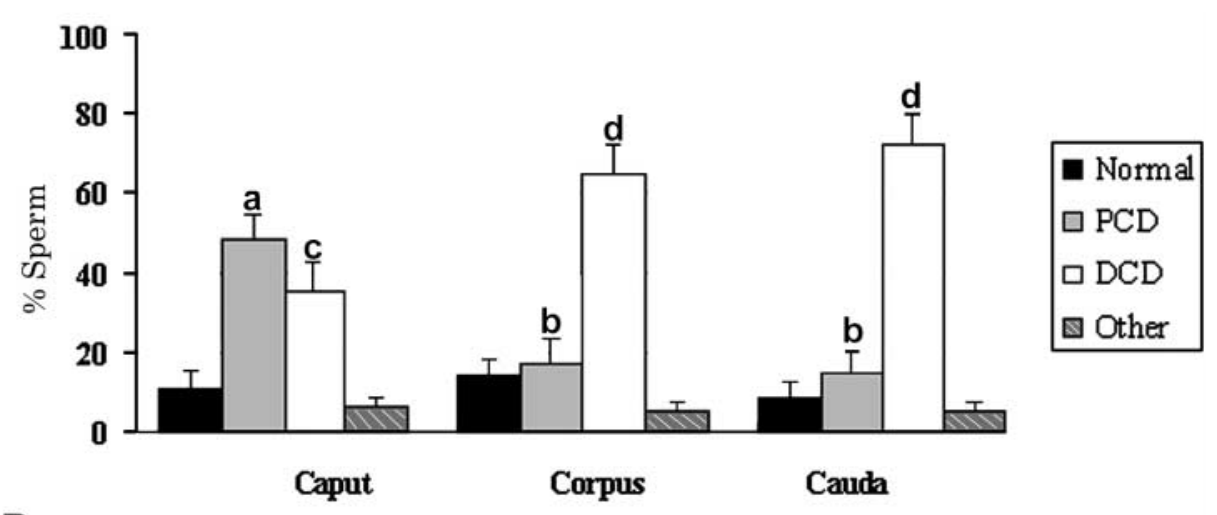

Figure 4 15-LOX is expressed in both testicular and epididymal spermatozoa during CD migration. (A) Western blotting of boar spermatozoa isolated from the testis (lane 1) and cauda epididymis (lane 2). Some degradation products of lesser molecular mass are seen in these preparations. (B) CD migration during boar sperm epididymal passage, as reflected by the comparisons of the least square means of different sperm populations in the caput, corpus and cauda epididymis. Average sperm populations from six boars were evaluated in the caput, corpus and cauda epididymis for morphologically normal spermatozoa (Normal), spermatozoa with proximal CD (PCD), spermatozoa with distal CD (DCD) and spermatozoa with abnormalities other than a CD (Other). Letters a-d indicate that values were significantly different at the $P<0.01$ level across epididymal compartments.

moved from the proximal to distal position during the passage between the caput and cauda epididymis (Fig. 4B). On average, more than $70 \%$ of cauda epididymal spermatozoa still contained distal CDs (Fig. $4 B$; the least square means for six boars are shown).

A boar with a high incidence (an average of $49.8 \%$ in five collections) of abnormal spermatozoa in ejaculates including, but not limited to, sperm-borne proximal and distal CDs, was identified in the course of the present studies. The boar was killed, and the testicular and epididymal spermatozoa from both sides of its reproductive tract were isolated and analyzed by light microscopy (Fig. 5A). Light microscopic sperm analysis determined that the right side of this boar's reproductive tract produced a significantly higher percentage of morphologically abnormal spermatozoa (abnormalities other than proximal or distal CDs) than the left side (Fig. 5A). While the sampling of spermatozoa from the left and right epididymis showed the migration of CDs to the distal position during epididymal passage, cauda epididymal spermatozoa on the right side still contained $20 \%$ of spermatozoa with gross morphological abnormalities other than distal $\mathrm{CD}$ retention (which is expected in $\sim 70 \%$ of the cauda epididymal spermatozoa in boar) (Fig. 5A). Interestingly, it appeared that the percentage of other visible morphological anomalies, while fairly constant on the unaffected left side, was reduced by half between caput and cauda epididymis on the right side (Fig. 5A). This observation indicates the epididymis has the capability to actively remove defective spermatozoa. Corresponding testicular and epididymal tissue samples from this boar were analyzed for the presence of 15-LOX by immunohistochemistry. Semi- niferous tubule degeneration accompanied by the increased accumulation of 15-LOX in the cytoplasmic lobes of elongated spermatids (Fig. 5C) was revealed by immunohistochemistry of the right testis while normal immunoreactivity of 15-LOX was seen in the left testis (Fig. 5B). Epididymal tissues from both sides (Fig. 5D and E) showed slight accumulation of 15-LOX in the cytoplasm of epididymal epithelial cells, and mainly on the apical surface of the epididymal epithelium, the site of both endocytosis and apocrine secretion of epididymal proteins. Such epididymal 15-LOX immunoreactivity, representative of epididymal tissues of the other five boars examined (not shown), was not observed in epididymal and testicular tissue sections incubated with pre-immune rabbit serum and appropriate secondary antibodies (Fig. 5F and G).

\section{Discussion}

It is widely recognized that the $C D$ migrates from the proximal to the distal position on the midpiece of the mammalian spermatozoa during sperm passage through the epididymis. In the present study, boar sperm CDs prevailed in a proximal position in the caput epididymis and then traveled down the midpiece of the spermatozoa between the caput and corpus epididymis. It is not clear when CDs are removed in the boar. Briz et al. (1995) reported droplet removal in the epididymis, while Harayama et al. (1996) suggested that components of seminal plasma, such as fructose or CAMP, might facilitate the completion of shedding of CDs from boar spermatozoa after ejaculation. The successful removal of CDs from 

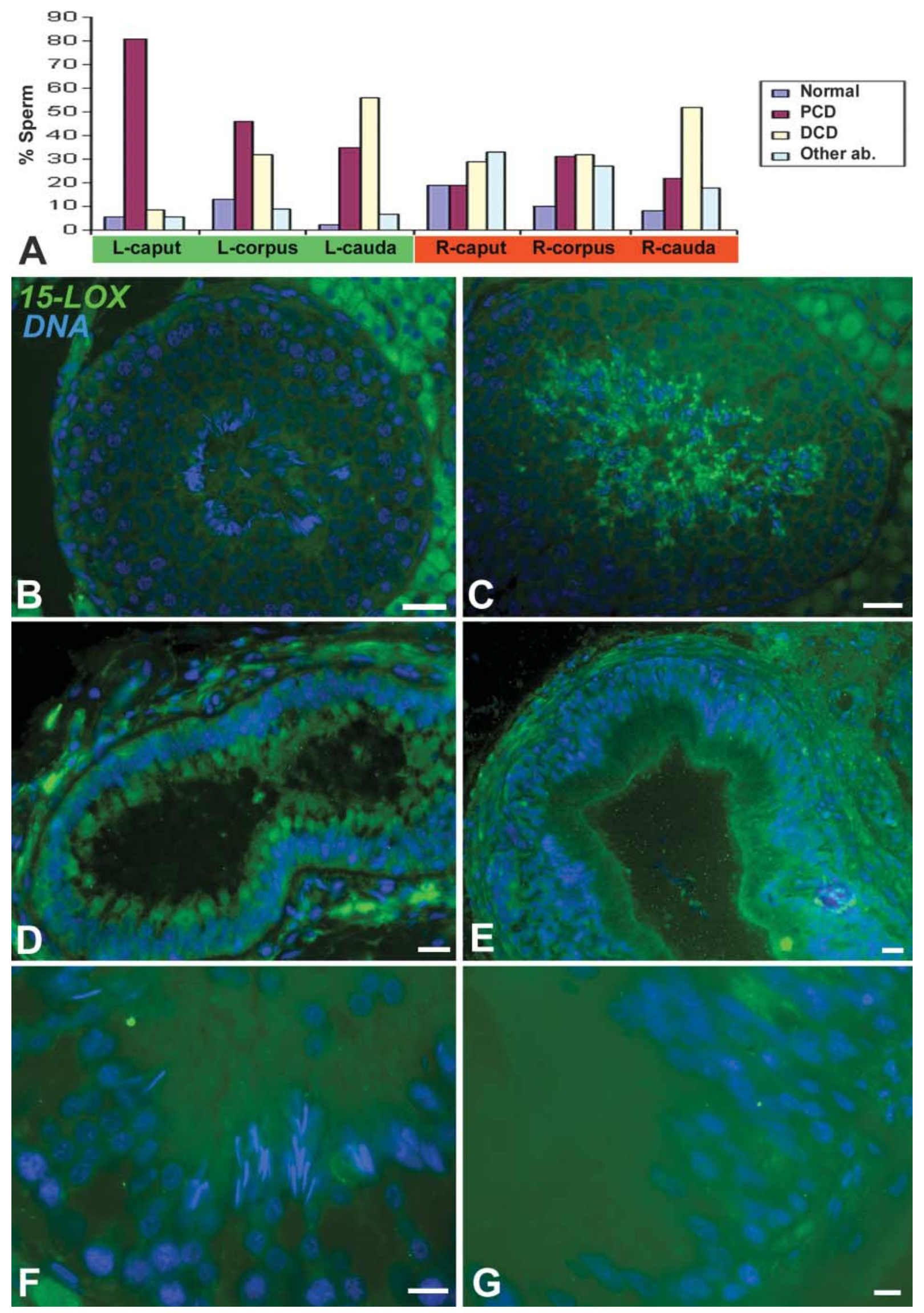
porcine spermatozoa may result from the synergy of the above mechanisms, but may also involve contributions from other pathways including the LOX and ubiquitin systems.

In this study, 15-LOX was found in the residual bodies rejected by elongating spermatids, and in the CDs of epididymal and ejaculated spermatozoa. 15-LOX was strongly associated with the fractions of the Percoll gradient that contained a large percentage of CDs, regardless of whether they were attached to the spermatozoa in the immotile sperm fraction or free in the debris fraction. The 15-LOX was not associated with normal, motile spermatozoa. This indicates that samples with a larger percentage of $C D$ retention on spermatozoa or in the debris fraction of the seminal plasma have stronger immunoreactivity to 15-LOX and 15-LOX is not abundant in motile spermatozoa without CDs.

In reticulocytes, 15-LOX is thought to be present both in the organelle membranes and on the inner face of the plasma membrane (Kühn et al. 1990). However, recent papers (van Leyen et al. 1998, Grüllich et al. 2001) have indicated that 15-LOX is present predominantly in reticulocyte organelle membranes, although some binding to the plasma membrane could be detected. Similarly to reticulocytes, the sperm CD contains organelle remnants destined for degradation, and the CD itself must be removed from a spermatozoon to eventually undergo degradation. The plasma membrane of the CD may become less rigid in structure after it has matured in the epididymis. Such a weakened plasma membrane on the mature CD may be prone to degradation, allowing 15-LOX substrates to be exposed that are normally protected in a non-damaged, immature CD plasma membrane. Similarly, isolated, processed plasma membranes used in studies described above may have exposed 15-LOX substrates due to processing. The 15-LOX enzyme could thus efficiently peroxidize both organelle membranes inside the $C D$ and the plasma membrane of the CD without damaging the sperm plasma membranes and membranes of the sperm mitochondrial capsule. In future experiments, specific inhibitors of 15-LOX activity could be used in an attempt to halt CD removal from isolated caput epididymal spermatozoa in an in vitro system.

While the presence of 15-LOX may contribute to the degradation of organelle membrane lipids in the CD, the mechanism for the degradation of the protein component of CD organelle membranes remains to be explained.
The present data and a recent report by Kuster et al. (2004) support a model in which the ubiquitin-dependent proteolytic pathway acts in synergy with the 15-LOX pathway for the degradation of the CD. This synergy could be similar to the respective contributions of the 15-LOX (van Leyen et al. 1998) and ubiquitin (Dubiel \& Rapoport 1989) pathways to reticulocyte organelle degradation. In the proposed model for organelle membrane degradation inside sperm CDs, the 15-LOX releases and/or degrades the lipid membrane components of the organelles in the CD and the ubiquitin-proteasome pathway is responsible for targeting the protein components of the CD for proteolytic degradation. Structurally altered by the respective activities of the 15-LOX and ubiquitin-proteasome pathways, the CD could descend from the connecting piece to the annulus, finally culminating in its release from the sperm axoneme to be liquefied and phagocytosed by the clear cells of the epididymal epithelium (Hermo et al. 1988) or rejected after ejaculation. Alternatively, 15-LOX could participate in epididymal sperm maturation and particularly in the morphogenesis of the sperm tail midpiece and mitochondrial sheath. We are currently investigating epididymal sperm maturation in a mutant mouse lacking the 15-LOX gene (ALOX mouse; Sun \& Funk 1996). Although the ALOX males are not infertile, our preliminary data indicate reduced sperm quality and altered $C D$ migration ( $\mathrm{K}$ W Lovercamp and P Sutovsky, unpublished observations).

Although in vitro fertilization trials were not performed for the present study, it should be considered that the 15LOX from superfluous CDs in boar seminal plasma could reduce the fertilizing potential of motile spermatozoa without CDs. A negative effect of infertile bull spermatozoa with retained CDs on normal bull spermatozoa was shown during bovine fertilization in vitro (Thundathil et al. 2001). In boars, the proportion of spermatozoa with distal CDs in an ejaculate had a negative correlation with pregnancy rates and litter size (Waberski et al. 1994). The accumulation of rejected CDs in semen could affect fertility directly by reducing sperm motility, or indirectly by releasing active enzymes and reactive oxygen species into seminal plasma (Aitken \& Clarkson 1988). Similarly, the soluble 15-LOX in semen could cause premature acrosomal exocytosis (Oliw \& Sprecher 1989, Lax et al. 1990), thus interfering with the process of sperm-zona binding and zona penetration. Altogether, the present study demonstrates that 15-LOX is a prominent enzymatic component of the sperm CD. It is to be determined if the

Figure 5 Relative numbers of abnormal spermatozoa and 15-LOX immunoreactivity within testicular and epididymal tissues of a boar with an unusually high occurrence of sperm defects in the ejaculate. (A) The relocation of the sperm CDs during epididymal passage, and an increased proportion of sperm abnormalities not related to $C D$ retention, as observed on the right side of reproductive tract of this boar. (B, C) Seminiferous tubule degeneration and increased content of 15-LOX in the cytoplasmic lobe of elongating spermatids were observed in the right testis (C), but not in the left testis (B). (D, E) There were no major differences in 15-LOX immunoreactivity between left (D) and right (E) epididymis (cauda sections are shown). All sections were processed concomitantly using a standard immunohistochemical procedure. (F, G) Negative control tissue sections of the boar testis and epididymis respectively (incubated with a non-immune rabbit serum and an appropriate fluorescent secondary antibody). Scale bar $=40 \mu \mathrm{m}$. Abbreviations, panel A: PCD = proximal CD; DCD $=$ distal CD; Other ab. $=$ other abnormalities; $\mathrm{L}$-caput $=$ left caput epididymis; R-caput $=$ right caput epididymis. Scale bars: $B-E=50 \mu \mathrm{m} ; \mathrm{F}, \mathrm{G}=10 \mu \mathrm{m}$. 
15-LOX present in the CD of boar spermatozoa is responsible for the degradation of $\mathrm{CD}$ organelle membranes and/or the removal of CDs from spermatozoa. Further studies will be pursued to determine the value of 15-LOX as an objective, quantitative marker of boar sperm quality by virtue of detecting differential amounts of 15-LOX in individual boar semen, thus reflecting the amount of both sperm-bound and free seminal CDs.

\section{Acknowledgements}

We thank Karen Bauman (Saint Louis Zoo) for providing babyrusa pig tissues, Dr Steve Terlouw and Brian Rojas (Premium Standard Farms) for domestic boar tissue and semen samples and Heinz Leigh for providing technical assistance. This work was supported by the USDA-NRI, Animal Reproduction Program (P S; award \#2002-02069), Food for the 21st Century Program of the University of Missouri-Columbia ( $\mathrm{P} \mathrm{S}$ ), and the Agricultural Experimental Station, University of Missouri-Columbia funding (T S). The authors declare that there is no conflict of interest that would prejudice the impartiality of this scientific work.

\section{References}

Aitken RJ \& Clarkson JS 1988 Significance of reactive oxygen species and antioxidants in defining the efficiency of sperm preparation techniques. Journal of Andrology 9 367-376.

Althouse GC 1998 Cytoplasmic droplets on boar sperm cells. Swine Health and Production 6128.

Baarends WM, Hoogerbrugge JW, Roest HP, Ooms M, Vreeburg J, Hoeijmakers HJ \& Grootegoed JA 1999 Histone ubiquitination and chromatin remodeling in mouse spermatogenesis. Developmental Biology 207 322-333.

Briz MD, Bonet S, Pinart B, Egozcue J \& Camps R 1995 Comparative study of boar sperm coming from the caput, corpus, and cauda regions of the epididymis. Journal of Andrology 16 175-188.

Dubiel W \& Rapoport SM 1989 ATP-dependent proteolysis of mitochondria of reticulocytes. Revisiones Sobre Biologia Celular 21 505-521.

Escalier D 2003 New insights into the assembly of the periaxonemal structures in mammalian spermatozoa. Biology of Reproduction $69373-378$.

Grüllich C, Duvoisin RM, Wiedmann M \& van Leyen K 2001 Inhibition of 15-lipoxygenase leads to delayed organelle degradation in the reticulocyte. FEBS Letters 489 51-54.

Harayama H, Shibukawa T, Miyake M, Kannan Y \& Kato S 1996 Fructose stimulates shedding of cytoplasmic droplets from epididymal boar spermatozoa. Reproduction, Fertility and Development 8 1039-1043.

Hermo L, Dworkin J \& Oko R 1988 Role of epithelial clear cells of the rat epididymis in the disposal of the contents of cytoplasmic droplets detached from spermatozoa. American Journal of Anatomy 183 107-124.

Kaplan M, Russell LD, Peterson RN \& Martan J 1984 Boar sperm cytoplasmic droplets their ultrastructure, their numbers in the epididymis and at ejaculation and their removal during isolation of sperm plasma membranes. Tissue and Cell 16 455-468.
Kühn H, Belkner J, Wiesner R \& Brash AR 1990 Oxygenation of biological membranes by the pure reticulocyte lipoxygenase. Journal of Biological Chemistry 265 18351-18361.

Kuster CE, Hess RA \& Althouse GC 2004 Immunofluorescence reveals ubiquitination of retained distal cytoplasmic droplets on ejaculated porcine spermatozoa. Journal of Andrology 25 340-347.

Lax Y, Grossman S, Rubinstein S, Magid N \& Breitbart H 1990 Role of lipoxygenase in the mechanism of acrosome reaction in mammalian spermatozoa. Biochimica et Biophysica Acta 1043 12-18.

National Animal Health Monitoring System Survey 2000 Part A: Reference of Swine health and management in the United States. In USDA National Animal Health Monitoring System. Fort Collins, USDA.

Oko R, Hermo L, Chan PT, Fazel A \& Bergeron JJ 1993 The cytoplasmic droplet of rat epididymal spermatozoa contains saccular elements with Golgi characteristics. Journal of Cell Biology 123 809-821.

Oliw EH \& Sprecher H 1989 Metabolism of polyunsaturated fatty acids by an (n-6)-lipoxygenase associated with human ejaculates. Biochimica et Biophysica Acta 1002 283-291.

Rapoport SM \& Schewe T 1986 The maturational breakdown of mitochondria in reticulocytes. Biochimica et Biophysica Acta 864 471-495.

Schewe T, Halangk W, Heibsch C \& Rapoport SM 1975 A lipoxygenase in rabbit reticulocytes which attacks phospholipids and intact mitochondria. FEBS Letters 60 149-152.

Sun D \& Funk CD 1996 Disruption of 12/15-lipoxygenase expression in peritoneal macrophages. Journal of Biological Chemistry 271 24055-24062.

Sutovsky P 2003 Ubiquitin-dependent proteolysis in mammalian spermatogenesis, fertilization, and sperm quality control killing three birds with one stone. Microscopy Research and Technique $6188-102$.

Sutovsky P 2004 Visualization of sperm accessory structures in the mammalian spermatids, spermatozoa and zygotes. Methods in Molecular Biology 253 59-77.

Sutovsky P, Moreno R, Ramalho-Santos J, Dominko T, Thompson WE \& Schatten G 2001 A putative, ubiquitin-dependent mechanism for the recognition and elimination of defective spermatozoa in the mammalian epididymis. Journal of Cell Science 115 5-7.

Thundathil J, Palasz AT, Barth AD \& Mapletoft RJ 2001 The use of in vitro fertilization techniques to investigate the fertilizing ability of bovine sperm with proximal cytoplasmic droplets. Animal Reproductive Science 65 181-192.

van Leyen K, Duvoisin RM, Englehardt H \& Wiedmann M 1998 A function for lipoxygenase in programmed organelle degradation. Nature 395 392-395.

Waberski D, Meding S, Dirksen G, Weitze KF, Leiding C \& Hahn R 1994 Fertility of long-term-stored boar semen influence of extender (Androhep and Kiev), storage time and plasma droplets in the semen. Animal Reproductive Science 36 145-151.

Wefes I, Mastrandrea LD, Haldeman M, Koury ST, Tamburlin J, Pickart CM \& Finley D 1995 Induction of ubiquitin-conjugating enzymes during terminal erythroid differentiation. PNAS 92 $4982-4986$.

Received 10 January 2005

First decision 18 March 2005

Revised manuscript received 14 April 2005

Accepted 23 May 2005 